\title{
El impacto y la percepción de los usuarios acerca del servicio del TransMicable en Bogotá D.C., como modo de última y primera milla
}

The Impact and the Perception of the Passengers About the Service of the TransMicable in Bogotá D.C., as a Last and First Mile Way Impacto e percepção dos usuários sobre o serviço TransMicable em Bogotá D.C., desde sua origem até seu destino final

Viviana Garnica Quiroga*

Recibido: 15 de noviembre del 2019

Aprobado: 4 de abril del 2020

https://doi.org/10.12804/revistas.urosario.edu.co/territorios/a.8456

Para citar este artículo:

Garnica Quiroga, V. (2021). El impacto y la percepción de los usuarios acerca del servicio del TransMicable en Bogotá D.C., como modo de última y primera milla. Territorios, (44), 171-184. https://doi. org/10.12804/revistas.urosario.edu.co/territorios/a.8456

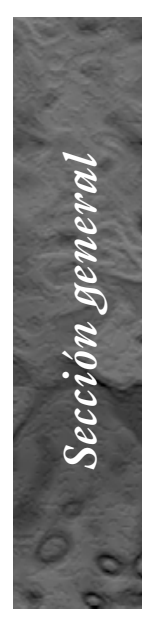

* Docente del programa de Ingeniería Civil, Universidad Piloto de Colombia. Correo electrónico:Viviana-garnica@ unipiloto.edu.co. ORCID: https://orcid.org/00000002-2867-8169. 
Palabras clave

Percepción;

transporte aéreo; última milla; usuario; viajes urbanos.

Keywords

Urban travel;

last mile;

air transport;

perception;

user.

Palavras-chave

Viagens urbanas; destino final; transporte aéreo; percepção; usuário.

\section{tersitarias 44}

\section{RESUMEN}

El transporte es una actividad fundamental en la cotidianidad de las personas. Es importante comprender que los viajes presentan varias etapas de desarrollo cuando se realizan en transporte público, y que cada etapa tiene una percepción distinta para el usuario. En territorios con condiciones topográficas montañosas y baja cobertura de transporte en corta longitud, es necesario desarrollar sistemas complementarios que faciliten la realización de los viajes. En ese sentido, en Bogotá fue construido un sistema aéreo denominado TransMicable, un servicio que cambió positivamente los patrones de viaje de los habitantes. Mediante la aplicación de encuestas a los usuarios del sistema se conoció la percepción en relación al cambio en la dinámica de los viajes de acceso y regreso al modo principal, se evidenció disminución en tiempos de viaje, en costos de transporte y mayor percepción de seguridad y calidad de vida. Así mismo, se encontró una favorabilidad al sistema por las mejoras urbanísticas y dinámicas sociales que ha traído consigo al área de influencia.

\section{ABSTRAC}

Transportation is a fundamental activity in people's daily lives. It is important to understand that trips have several stages of development when they are in public transportation, and each stage has a different perception for the user. In territories with a highland topography and low coverage of short-length transport, it is necessary to develop complementary systems that facilitate travel. In the city of Bogotá, an air system called TransMicable was built; this system positively changed the travel patterns of the people in the area. Surveys were done to the people of the area and the perception regarding the dynamics of the trips was known. The decrease in travel time and cost was evidenced, and the mayor perception was regarding safety and quality life. Moreover, it was shown that the system brought an urban improvement and new social dynamics to the area.

\section{RESUMO}

O transporte é uma atividade fundamental no cotidiano das pessoas. É importante compreender que as viagens apresentam várias etapas de desenvolvimento quando se realizam por transporte público, pois cada etapa tem uma percepção distinta para o usuário. Em território com topografia montanhosa e baixas opções de transporte para trajetos curtos, se faz necessário o desenvolvimento de sistemas complementares que facilitem a realização dessas viagens. Na cidade de Bogotá foi construído um sistema aéreo chamado TransMicable, que mudou positivamente os padrões de viagem dos habitantes. Através da aplicação de pesquisas de opinião com os usuários do sistema, conheceram-se suas percepções em relação à mudança nas viagens de ida e volta à modalidade principal, também se evidenciou a redução na duração da viagem, nos custos de transporte e maior percepção de segurança e qualidade de vida. Ainda assim, se evidenciou favorecimento do sistema em relação às melhoras urbanísticas e dinâmicas sociais que o mesmo trouxe consigo ao impactar na área em que está inserido. 


\section{Introducción}

Para analizar los viajes en transporte público, es necesario entender el comportamiento del usuario desde el punto inicial (origen) hasta el punto final o de destino. En tal sentido, como lo menciona Meng et al. (2018), un viaje urbano en transporte público inicia con el tiempo de acceso o primera milla, tiempo de espera, tiempo de viaje, tiempo de trasbordo - si es necesario-, nuevo tiempo de viaje y finalmente tiempo de regreso o última milla. Alonso Ramos et al. (2012), mencionan que ningún viaje de largo recorrido consta de una sola etapa - a menos que se realice en vehículo particular-, pues sea de acceso o regreso estos recorridos pueden en ocasiones determinar el viaje principal.

Agarwal et al. (2019), indican que la planeación separada de los modos de transporte en cada una de las etapas de los viajes no permite la integración del servicio, lo cual genera que el transporte público no sea atractivo para los usuarios y migren a otros sistemas que generan mayores externalidades al territorio. Para que el transporte público sea competitivo es necesario que los tiempos de operación estén coordinados - minimizar tiempos de espera/trasbordo-, exista integración física y tarifaria, buenas condiciones vehiculares y, que indiscutiblemente tenga la conexión entre el origen y el destino. Zellner et al. (2016), confirman que cuando la conexión entre la parada final del sistema de transporte público y el punto de destino es muy distante, es poco probable que la alternativa elegida sea dicho sistema. Ya que precisamente, es esa conexión inicial o final la que es fuertemente castigada por los usuarios tanto en tiempo como monetariamente. Agarwal et al. (2019), estiman que la última milla se percibe como la milla más larga dentro del viaje global. Cuando esta última milla se realiza en modo a pie, un pasajero percibe que está caminando durante 10743 minutos por cada minuto que realmente camina. Además, si el clima es malo percibe que está caminando 0,2069 minutos adicionales (Meng et al., 2018). Para los planificadores de transporte, esta última milla se torna compleja por efectos del entorno social, la demanda y el costo. Tilahun et al. (2016), indican que el problema de la última milla es multidimensional porque tiene componentes físicos — basados en el lugarsociales y perceptivos; en suma, abordar este problema requiere un enfoque múltiple que consiste no solo en soluciones de transporte y diseño urbano, sino también en políticas sociales amplias.

Es este vacío el que permite la aparición de diversos tipos de sistemas de transporte alternativo formalizado o no - minibuses, camionetas, taxis, vehículos de tres ruedas y motocicletas que manejan ilícitamente su comercio en muchas ciudades-. Los operadores de pequeña escala ingresan al mercado para llenar estos vacíos, complementan los servicios de tránsito regulares, ingresan a los barrios mal atendidos por 
Figura 1. Ubicación localidad Ciudad Bolívar

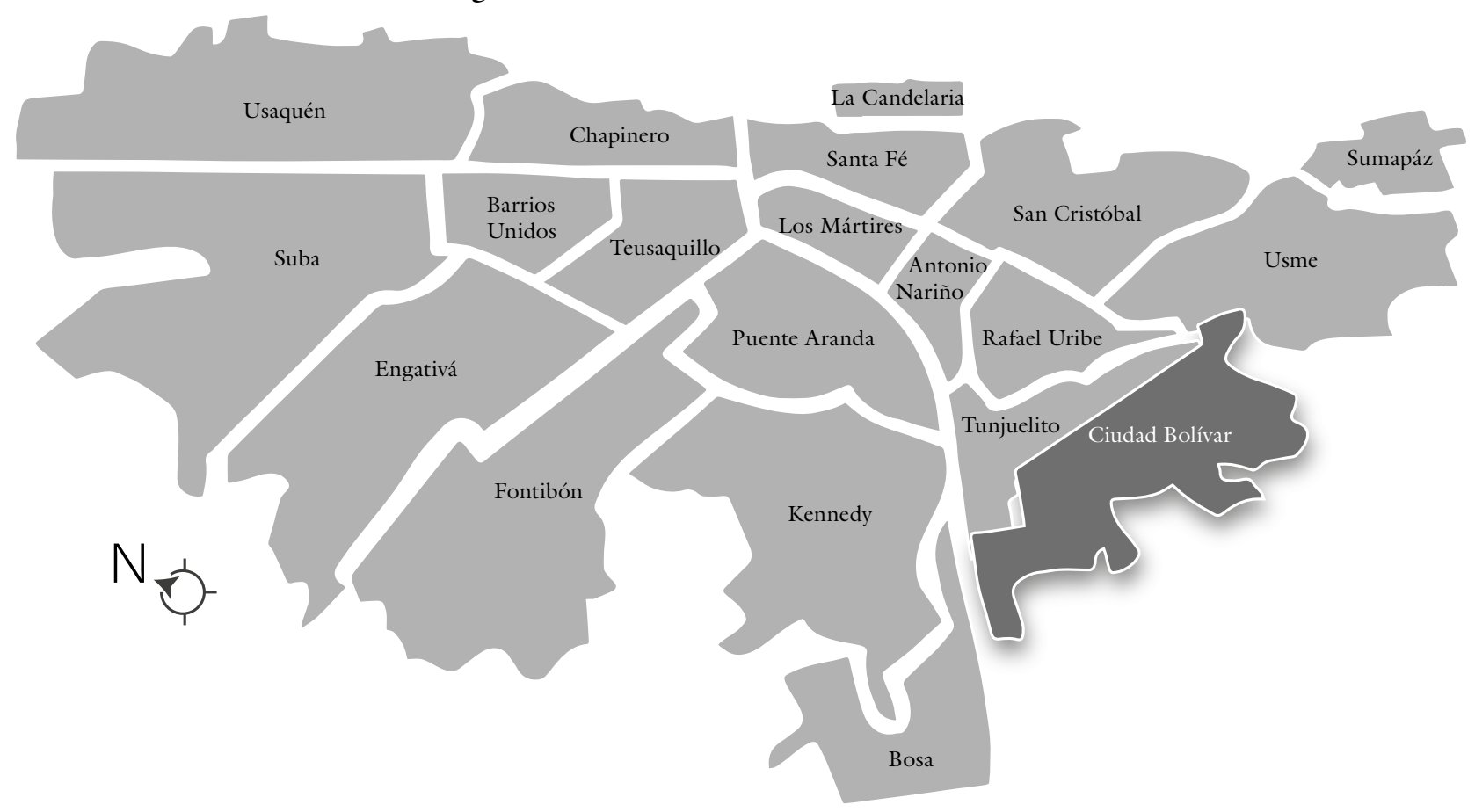

Fuente: Secretaría de integración Social (2018).

los operadores formales y responden rápidamente a las cambiantes demandas del mercado (Cervero \& Golub, 2007).

Sin embargo, en muchas ciudades, en especial aquellas con altas pendientes topográficas, se han implementado sistemas aéreos que complementan los principales sistemas de transporte y mejoran la movilidad urbana —en la primera y última milla-, entre ellas: Portland, Oregón (EE. UU.); Roosevelt Island, Nueva York (EE. UU.); Medellín (Colombia); Caracas (Venezuela); Hong Kong (China); Lagos
(Nigeria); Constantino (Argelia); Río de Janeiro (Brasil); Coblenza (Alemania); Mao Kong (Taiwán); y La Paz-El Alto (Bolivia).

\section{Contexto}

Ciudad Bolívar es una de las localidades más extensas de Bogotá, está ubicada al sur de la ciudad (13000 hectáreas) como se muestra en la figura 1 y cuenta con ocho Unidades de Planeamiento zonal (UPZ) en su territorio urbano. Se caracteriza por 
ser una zona montañosa en un $90 \%$ - la zona urbana más baja de la localidad está ubicada a una altitud de $2400 \mathrm{msnm}$ y la

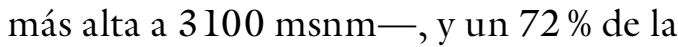
localidad se considera área rural (Alcaldía Local de Ciudad Bolívar, 2018).

La distribución poblacional es del $11,8 \%$ para la primera infancia ( 0 a 5 años), 15,4\% infancia (6 a 13 años), 7,6\% adolescencia (14 a 17 años), 15,7\% juventud (18 a 26 años), 41,8\% adultez (27 a 59 años) y 7,8\% vejez (mayores de 60 años). Para el 2015 se identificó que el $4 \%$ de la población de la localidad eran personas pertenecientes a población diferencial, de esta población el $88,8 \%$ era víctima de desplazamiento, $5,2 \%$ se encontraba en condición de discapacidad, 4,8\% tenían alguna pertenencia étnica, $1 \%$ eran habitantes de calle y $0,2 \%$ eran población LGBTI. La mayor parte de la población es de estratos socioeconómicos 1 y 2 -entiéndase estrato socioeconómico como un indicador de ingresos al hogar-.

De acuerdo con la Encuesta de Movilidad 2015, el modo de desplazamiento principal de los habitantes de Ciudad Bolívar es caminando con un $41,9 \%$, de los más de 899700 viajes que se realizan a diario en dicha localidad. Luego, se encuentran los trayectos en el servicio urbano del SITP que representan el 26,6\% de los viajes, TransMilenio se utiliza en $12,4 \%$ y los desplazamientos en motocicleta son el $4,4 \%$ de los viajes.

Moyano Bello et al. (2018), indican que en Ciudad Bolívar más del $60 \%$ de la localidad no tiene vías y medios de transporte para comunicarse con el resto de la ciudad, por lo cual los viajes en automóvil representan un porcentaje marginal dentro de los modos posibles. Así mismo, el uso de bicicleta es bajo $(2,9 \%)$ y se asocia a las pronunciadas pendientes del territorio. Se estima que un recorrido medio en un motorizado entre la parte alta de la localidad hasta el centro de la ciudad tarda al menos 2 horas y, el costo de esa movilización puede llegar a los $\$ 5000$ pesos por trayecto. En ese marco, Amézquita et al. (2016) indican que por razones como las mencionadas, se incentiva a largas caminatas y a la aparición de transporte alternativo poco regulado.

A raíz de esta problemática, para el 2018, en alianza entre la Corporación Andina de Fomento (CAF), TransMilenio, Cable Móvil e Ilunion Tecnología y Accesibilidad, se concluyó la construcción y puesta en marcha del primer teleférico de transporte público de Bogotá en Ciudad Bolívar. Este sistema se caracteriza por la instalación de transporte en la que los vehículos están suspendidos de uno o más cables. En este caso, los vehículos son de tipo telecabina - cerrados y de baja capacidad-. TransMicable en conjunto con el componente troncal y zonal conforman el Sistema Integrado de Transporte Público de la ciudad de Bogotá (SITP). La extensión del sistema es de $3,34 \mathrm{~km}$ de longitud y es una alternativa para recorrer la primera y última etapa de algunos viajes en la localidad de Ciudad Bolívar. Del 
Figura 2. Localización de estaciones sistema TransMicable, Ciudad Bolívar

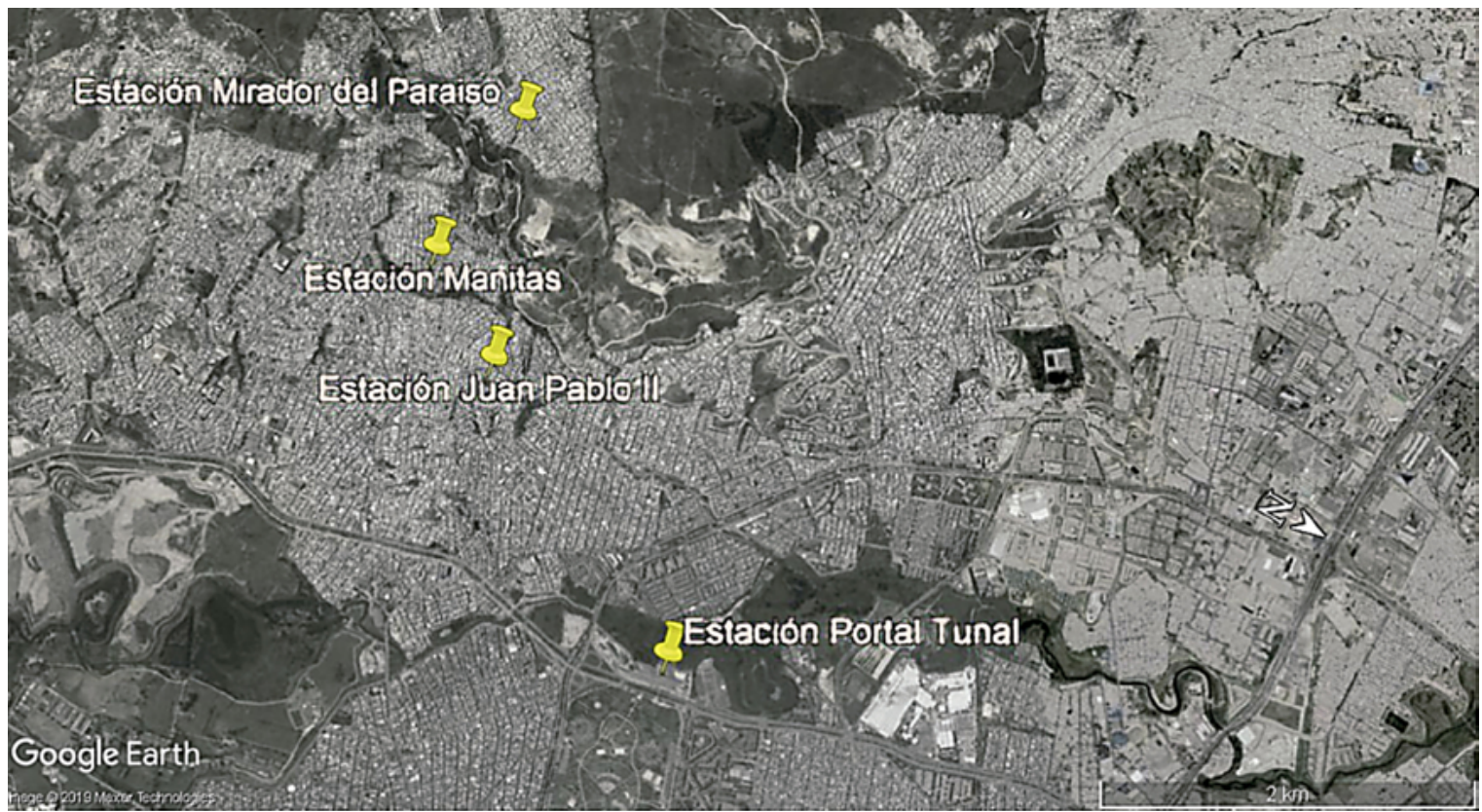

Fuente: elaboración propia a partir de Google Earth (2019).

territarias 44 sistema se espera una capacidad horaria de 3600 pasajeros por sentido por hora (TransMilenio S.A., 2018).

Este sistema se encuentra conectado física y tarifariamente con el componente troncal de la ciudad en el Portal Tunal, lo que facilita la intermodalidad en el desarrollo de los viajes de los 80000 habitantes del área de influencia. El proyecto integró tres elementos significativos: el primero, la infraestructura del cable cuyo costo representó \$240000 millones de pesos; el segundo, la renovación urbana en las zonas aledañas cuya inversión fue de alrededor $\$ 134000$ millones de pesos; y el tercero, el trabajo con comunidad, gestión social y cultura ciudadana (Alcaldía Mayor de Bogotá, 2018).

La ubicación de las estaciones se muestra en la figura 2, en la cual se evidencia que el mayor impacto se presenta en la UPZ El Lucero, cuya población para el 2017 fue de 196259 (Consejo Local de Gestión del Riesgo y Cambio Climático, CLGR-CC, 2019). El sistema inicia en el Portal Tunal de TransMilenio, y se eleva a $2827 \mathrm{msnm}$ hacia los barrios Paraíso y Mirador. Esta ubicación solo genera cobertura en una parte de la localidad, el área restante aun presenta dificultades de transporte. 
El horario de funcionamiento del sistema es de lunes a sábado desde las 4:30 a.m. hasta las 10:00 p.m., y los domingos y festivos de 5:30 a.m. a 9:00 p.m. Este sistema no solo trajo a la comunidad una mejora en los tiempos de desplazamiento, sino que además aumentó el índice de empleabilidad, puesto que la política del sistema es vincular a los habitantes del sector en la operación de este.

En este artículo se busca determinar cuál es la percepción de los usuarios en el área de influencia a partir de la inclusión de este sistema, y cuál ha sido el efecto en la etapa de inicio y fin de los viajes principales.

\section{Materiales y métodos}

Al reconocer la importancia de la calidad esperada en la información a tomar en campo, la herramienta que se utilizó - en este caso una encuesta de interceptación directa-, fue probada en varios escenarios piloto (laboratorio de urbanismo de la Universidad Piloto de Colombia). En este espacio se encuestó a una muestra reducida de personas con la finalidad de evaluar la congruencia del diseño, junto con la identificación de puntos que podrían generar confusión en los encuestados al momento de responder las preguntas. De la prueba piloto se realizaron ajustes al diseño inicial, hasta llegar a la versión final cuyos acápites se mencionan a continuación. La encuesta consta de 3 apartes significativos:
- Parte 1: en esta parte inicial se generó la caracterización de los viajes actuales $\mathrm{y}$ anteriores al desarrollo del sistema tanto en la ida como en el regreso, se tuvo en cuenta el origen, destino, motivo de viaje, modo de acceso al sistema y tiempos de desplazamiento en la ida y en el regreso.

- Parte 2: en la parte media se indagó respecto a la percepción del TransMicable y el impacto que ha tenido en el territorio en aspectos de infraestructura, seguridad social y aspectos económicos.

- Parte 3: por último, se generó la caracterización sociodemográfica del encuestado.

Las encuestas se realizaron en junio y agosto del 2019, al interior del sistema y en áreas aledañas a las diferentes estaciones, la duración máxima de cada encuesta fue de alrededor 11 minutos.

El motivo por el cual se realizaron dentro del sistema fue para generar recordación del contexto al usuario, así mismo, como la mayoría de los viajes en el sistema se realizan desde la estación El Paraíso hasta el Portal Tunal y viceversa, el tiempo era justo para contestar dicho instrumento.

En total se realizaron 280 encuestas mediante interceptación directa. Teniendo en cuenta que el sistema reporta 21000 pasajeros en los días de máxima demanda (Martínez, 2019), se estableció de acuerdo con el cálculo de muestra para poblaciones territarias 44 
finitas, un muestreo de 269 encuestas para un nivel de confianza del $90 \%$ y margen de error del $5 \%$.

Para complementar esta información, en reuniones focales con la comunidad aledaña al sistema, se realizaron una serie de conversatorios libres con apoyo de los líderes y lideresas sociales. Esta actividad permitió profundizar en la percepción que se tenía respecto a la implantación del sistema como posibilidad para realizar los viajes de acceso o regreso en los desplazamientos.

\section{Resultados}

De acuerdo con la información analizada se establece que la muestra tomada de encuestas es representativa para la población de estudio. Se destaca la participación del género femenino en un $52,7 \%$, dentro de las cuales la mayoría se encuentra entre los 18 y 30 años. En el género masculino, la representación de las personas jóvenes es igualmente representativa con un $34,8 \%$, tal como se muestra en la figura 3. Esta información evidencia que existió una cobertura idónea en cuanto a género y edad de la población y, no se generó sesgo alguno en estos aspectos. Adicionalmente permite tener una caracterización sociodemográfica básica de los usuarios del sistema.

Como se puede ver en la figura 4 , la mayoría de los usuarios del sistema $(77,2 \%)$ reciben ingresos inferiores a $\$ 1000000$ de los cuales el $35,4 \%$ son empleados $\mathrm{y}$, en menor proporción se encuentran

Figura 3. Distribución de género y edad de la muestra del estudio

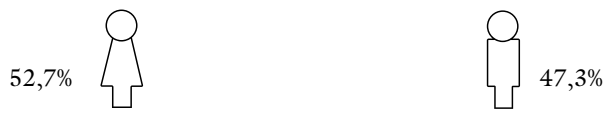

Menos de 18 años

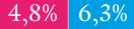

Entre 18 y 30 años \begin{tabular}{l|l|l}
$44,8 \%$ & $34,8 \%$
\end{tabular}

Entre 31 y 40 años $20,8 \% \quad 18,8 \%$

Entre 41 y 50 años \begin{tabular}{l|l}
$15,2 \%$ & $15,2 \%$
\end{tabular}

Entre 51 y 60 años

\begin{tabular}{l|l}
$8,8 \%$ & $16,1 \%$ \\
\hline
\end{tabular}

Más de 60 años

$5,6 \% \quad 8,9 \%$ 
Figura 4. Caracterización económica usuarios de TransMicable

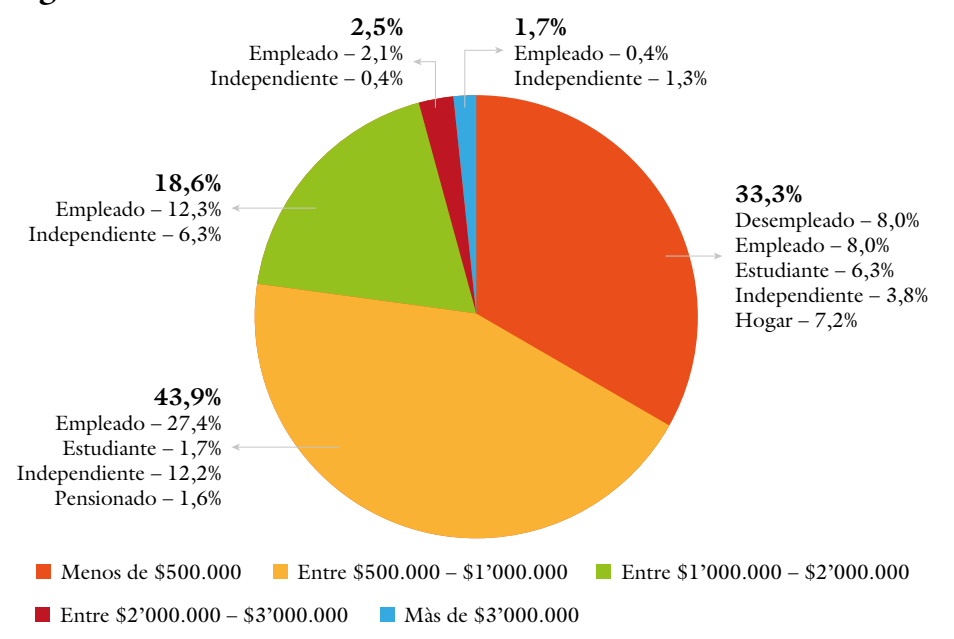

Fuente: elaboración propia (2019).

trabajadores independientes, estudiantes, pensionados, personas dedicadas al hogar y desempleados. Este resultado comprueba que la necesidad de un servicio de transporte público con calidad y equilibrio en las tarifas es latente, puesto que el porcentaje de gasto en los ingresos asociados al transporte debe permitir la cobertura de otras necesidades primarias para garantizar calidad de vida en los habitantes.

El motivo de viaje de la mayoría de los usuarios del sistema es laboral $(59,1 \%)$, seguido de otros - diligencias, compras, recreación, salud-y estudio. Para estos viajes, el modo principal no es el TransMicable, pero este sistema sí hace parte del inicio o el fin de algún trayecto. Como se observa en la figura 5 , los tiempos de viaje evaluados en un solo trayecto (ida) disminuyeron considerablemente a partir de la puesta en operación del sistema Transmicable. Se establece que antes de TransMicable los viajes eran superiores a 15 minutos y alcanzaban tiempos de hasta 150 minutos (más de 2 horas), escenario que mejoró a partir de la operación y muestra de este sistema. Los viajes con menos de 15 minutos se redujeron en un $8,7 \%$ y los viajes de más de 106 minutos se redujeron en $26,7 \%$. Como se ha mencionado, la calidad de vida con la disminución del tiempo de viaje se ha mejorado, puesto que al tratarse la mayor parte de los viajes por motivo laboral, se calcula que como mínimo se realizan cinco días a la semana, y al acumular el tiempo ahorrado podría inferirse la realización de otras actividades o de descanso en sí mismo. 
Figura 5. Motivo y tiempo de viaje usuarios de TransMicable

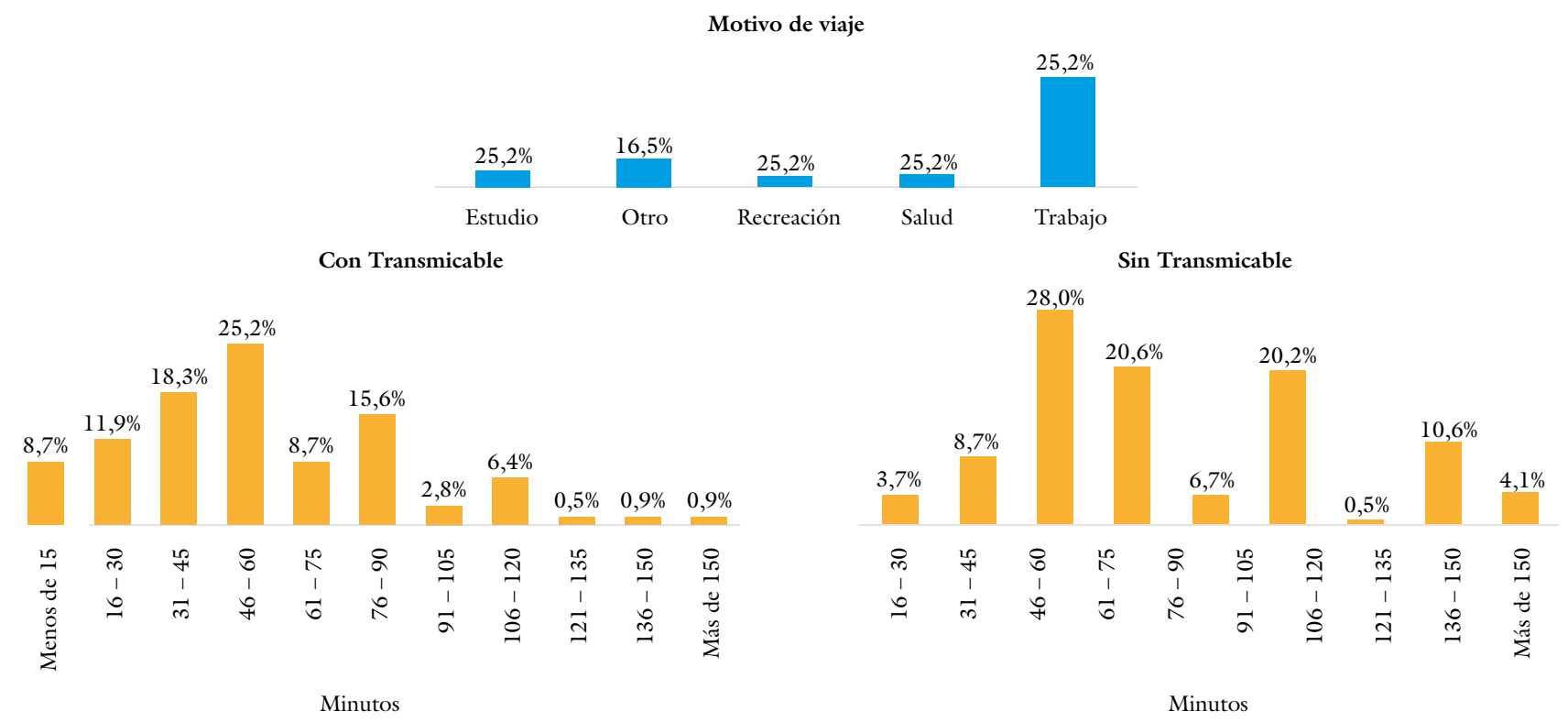

Fuente: elaboración propia (2019).

\section{territarias 44}

Este escenario no se presenta en totalidad para el regreso de los usuarios, el $15,6 \%$ de los viajeros no usa el sistema TransMicable en los viajes de regreso. Tal comportamiento es derivado del posible incremento en el costo del viaje asociado a la posibilidad que tiene un usuario de cambiar de sistema sin costo de transbordo que, para este caso es de 75 minutos, adicionalmente la operación de TransMicable termina antes que la del sistema de buses de tránsito rápido (BRT). Por lo anterior, los usuarios migran a otros sistemas en el viaje de regreso. Como ejemplo, se puede referir un usuario que tenga su regreso desde el Portal Suba en el noroccidente de la ciudad hasta El Mirador el Paraíso, mediante la consulta en diferentes aplicaciones tecnológicas que funcionan en la ciudad, se establece que desde que aborda el bus del sistema en el portal de origen, únicamente hasta el portal el Tunal tendrá una duración media de 89 minutos —asumiendo que no requiere transbordo-, sin embargo, este tiempo podría incrementar por efectos de congestión y otros. En este ejemplo se evidencia que no es posible acceder al TransMicable sin que se haya validado de nuevo una tarifa plena, por lo cual muchos usuarios en el regreso, según este escenario, migran al sistema alimentador 
que no refleja costo monetario alguno. Se muestra que por trayecto el 79,2\% de los usuarios gastan alrededor de $\$ 3000$ pesos y está sujeto a las condiciones del tráfico y de la demanda de los sistemas, para alcanzar o no el trasbordo sin costo alguno.

Ahora bien, aunque el sistema ha mejorado la calidad del transporte para los habitantes de Ciudad Bolívar, este no significa ser el primer o último modo de los viajes. En la figura 6 se observan los modos de conexión del sistema, se muestra que tanto para el regreso y acceso a este predomina el modo a pie o caminata y que los modos motorizados también hacen presencia con menor participación. El uso de alimentador y del componente zonal se realiza cuando existe la cobertura de los servicios para realizarlo. Sin embargo, aún existen zonas con baja o nula cobertura de transporte público integrado, lo cual desencadena la aparición de sistemas alternativos como los vehículos particulares de pasajeros o la utilización de plataformas tecnológicas como Uber, Beat, Didi, entre otros. El uso de vehículos particulares que de manera ilegal transportan pasajeros, predomina sobre el de las App por el costo asociado, esta utilización prevalece en el regreso o finalización de los viajes.

En general, la percepción de los usuarios de TransMicable es positiva en un $92,7 \%$, ya que este sistema ha mejorado

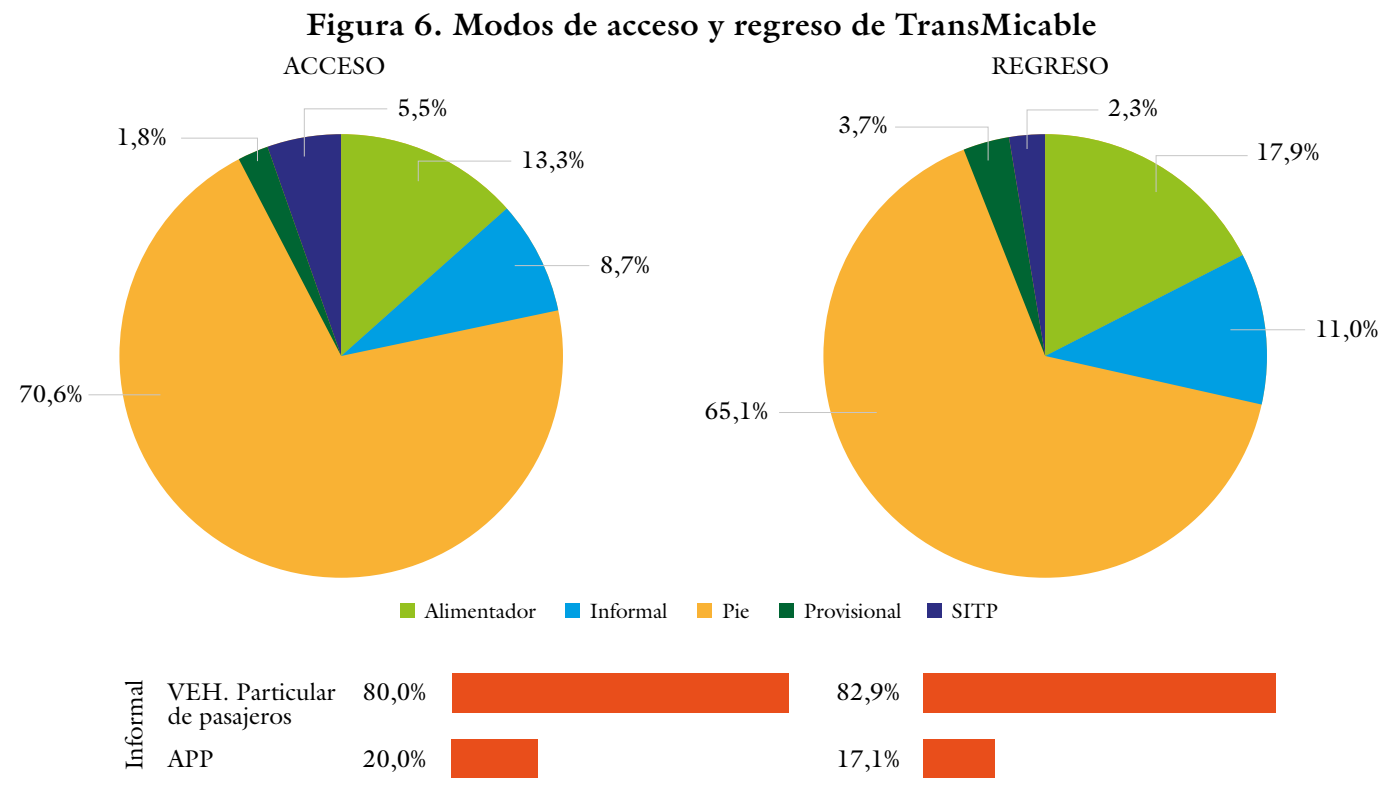

Fuente: elaboración propia (2019). 
la conexión inicial o final del modo principal de los viajes cotidianos. De igual manera, el 89,0\% percibe más seguridad tanto física como social y, el 91,3\% siente que su calidad de vida ha mejorado desde entonces. Adicionalmente, la percepción de la calidad del servicio es alta, y se mencionan como aspectos relevantes la garantía de accesibilidad para todas las condiciones físicas y la fiabilidad en la orientación para el uso por parte de los funcionarios del sistema.

\section{Discusión y conclusiones}

Dado que el objetivo del presente estudio fue encontrar la percepción y el impacto que han sentido los usuarios del sistema TransMicable de Bogotá como medio de conexión al modo principal de viaje, los resultados hacen evidente que el desarrollo de sistemas que faciliten los desplazamientos de última o primera milla es una necesidad vigente en muchas ciudades. Este es un tema que debe evaluarse desde la planificación del territorio, que permita garantizar accesibilidad completa a las personas, no se debe olvidar que el transporte es una actividad derivada de otras actividades sociales como el estudio, la salud, el comercio, el trabajo, entre otros, por lo cual se hace fundamental. Así mismo, se ha evidenciado que la falta de este tipo de sistemas favorece la aparición y durabilidad de sistemas alternativos, formalizados o no, en especial en zonas con topografía agreste o de difícil acceso.
La implantación de TransMicable en la localidad de Ciudad Bolívar ha traído consigo mejor calidad de vida a los habitantes, no solo asociado a la disminución en los tiempos de viaje sino también al despliegue de infraestructura dotacional que se ha construido en torno al sistema y, las campañas de gestión social que se han realizado promoviendo cultura de cuidado y conservación. Además, ha existido una renovación urbana en cuanto a mejora de fachadas, andenes y espacio público en general, así como muestras artísticas en murales y tejados de las viviendas del sector.

La percepción de seguridad y calidad del transporte también ha incrementado, y esto se asocia a la integración física del sistema con la operación de TransMilenio — modo principal de viaje en Bogotá- y a la operación propia — velocidad, comodidad, información y personal de apoyo-. Sin embargo, el tiempo posible de trasbordo para los viajes con largas distancias se hace corto, sobre todo en horas de máxima demanda, este comportamiento hace que se aumente el costo del trayecto o que se migre a modos de menor costo. De manera adicional, el horario de funcionamiento es menor que el del sistema TransMilenio y este es otro factor que influye en la utilización del sistema. En general, el panorama de percepción de los usuarios es positivo y se espera dé continuidad al desarrollo de más sistemas de este tipo en la ciudad. Por último, vale la pena mencionar que este trabajo es el inicio de una propuesta 
de investigación en la Universidad Piloto de Colombia, denominada "Movilidad urbana y el entorno, como generadores de ciudadanía”, que busca con fases posteriores responder: ¿Cómo la conformación del territorio en una ciudad como Bogotá condiciona de manera directa la forma de desplazamiento y las prácticas de la población en torno a la movilidad cotidiana y su entorno como generador de ciudadanía?

\section{Agradecimientos}

A la Universidad Piloto de Colombia por el apoyo en el despliegue de la investigación, a la empresa de TransMilenio por permitirnos acceder a la operación del sistema y tomar las encuestas, y a los líderes de diferentes comunidades de Ciudad Bolívar, quienes participaron en la investigación y nos contaron sus experiencias de transporte.

\section{Referencias}

Agarwal, O. P., Kumar, A., \& Zimmerman, S. (2019). Network planning: from segment to whole trip. In Emerging paradigms in urban mobility. Planning, financing and management (pp. 101-122). Elsevier. https://doi. org/10.1016/B978-0-12-8114346.00005-6

Alcaldía Mayor de Bogotá. (2018). Proyecto integral transMicable. Documento de Sistematización de Proyecto Integral Transmicable.pdf. https:// secretariageneral.gov.co/sites/default/ files/planeacion/20181231_informegestionresultados_2018_0_0.pdf

Alcaldía Local de Ciudad Bolívar. (2018). Conociendo mi localidad. Alcaldía Mayor de Bogotá. http://www. ciudadbolivar.gov.co/mi-localidad/ conociendo-mi-localidad/historia

Alonso Ramos, A., Monzón de Cáceres, A., \& López-lambas, M. E. (2012). A vueltas con la última milla. Universidad Politécnica de Madrid. http://oa.upm.es/31154/1/INVE_MEM_2014_176254.pdf

Amézquita, L., Durán Matiz, D., \& Fajardo Morales, D. (2016). Matriz origen-destino y eficiencia en modos de transporte urbano: un análisis de la movilidad de Bogotá. Semestre Económico, 19(39), 91-111. https://doi. org/10.22395/seec.v19n39a4.

Cervero, R., \& Golub, A. (2007). Informal transport: A global perspective. Transport Policy, 14(6), 445-457. https://doi.org/10.1016/j.tranpol.2007.04.011

Consejo Local de Gestión del Riesgo y Cambio Climático (CLGR-CC) de Ciudad Bolívar. (2019). Plan Local de Gestión del Riesgo y Cambio Climático PLGRCC CB. Alcaldía Mayor de Bogotá. https://tinyurl.com/y5zrbh28

Martínez, J. L. (2019). TransMicable ha transportado a 1.8 millones de pasajeros. Alcaldía Mayor de Bogotá. https://bogota.gov.co/mi-ciudad/ movilidad/pasajeros-de-transmicable 
Meng, M., Rau, A., \& Mahardhika, H. (2018). Public transport travel time perception: Effects of socioeconomic characteristics, trip characteristics and facility usage. Transportation Research Part A: Policy and Practice, 114(January), 24-37. https://doi. org/10.1016/j.tra.2018.01.015

Moyano Bello, N. J., Prieto, A. D., \& Martínez Guevara, F. M. (2018). Evaluación de los impactos sociales proyectados para transMicable en torno a la Estación Juan Pablo II de la Localidad Ciudad Bolivar. Universidad Católica de Colombia. https://hdl.handle. net/10983/22383

Secretaría de Integración Social. (2018). Mapa localidades Bogotá. Alcaldía Mayor de Bogotá. https://www.integracionsocial.gov.co/index.php/ entidad/informacion-institucional/ localidades-sdis/38-entidad/localidades

Tilahun, N., Vonu, P., Li, M., \& Keita, Y. (2016). Transit use and the work commute: Analyzing the role of last mile issues. Journal of Transport Geography, 54, 359-368. https://doi. org/10.1016/j.jtrangeo.2016.06.021

Transmilenio S.A. $(2018,23$ de diciembre). Abecé de TransMiCable. https:// www.transmilenio.gov.co/publicaciones/151067/abece-de-transmicable/

Zellner, M., Massey, D., Shiftan, Y., Levine, J., \& Arquero, M. J. (2016). Overcoming the last-mile problem with transportation and land-use improvements: An agent-based approach. International Journal of Transportation, 4(1), 1-26. https:// doi.org/10.14257/ijt.2016.4.1.01 